\title{
GUAYALEJO, VARIEDAD DE CÁRTAMO PARA LA REGIÓN DE LAS HUASTECAS
}

\author{
GUAYALEJO, A SAFFLOWER VARIETY FOR THE HUASTECAS REGION
}

\author{
Juan Valadez-Gutiérrez ${ }^{1 *}$ y José E. Cervantes-Martínez ${ }^{2}$
}

\begin{abstract}
'Campo Experimental Las Huastecas, Instituto Nacional de Investigaciones Forestales Agrícolas y Pecuarias (INIFAP). Km 55 Carretera TampicoMante. 89610, Villa Cuauhtémoc, Municipio de Altamira, Tamaulipas. Tel. 015538718700 Ext. 83302. ²Facultad de Agronomía, Unidad Académica Multidisciplinaria Mante. Universidad Autónoma de Tamaulipas.
\end{abstract}

*Autor para correspondencia (valadez.juan@inifap.gob.mx)

En respuesta a las demandas de los productores de la región de las Huastecas (norte de Veracruz, oriente de San Luis Potosí y sur de Tamaulipas), en el Instituto Nacional de Investigaciones Forestales, Agrícolas y Pecuarias (INIFAP) se obtuvo la variedad de cártamo (Carthamus tinctorious L.) Guayalejo de alto potencial de rendimiento y estabilidad en su producción de grano. Esta variedad es tolerante a las enfermedades principales en esa región: Mancha de la hoja (Alternaria carthami) y Roya (Puccinia carthami); contiene $35.64 \%$ de aceite, con una proporción linoleico:oleico de 3:1 (Aceites especiales TH, abril 17 de 2015)'. En el Catálogo Nacional de Variedades Vegetales 2015, a esta variedad se le asignó el número de registro definitivo CAR-019-230513 (SNICS, 2015).

Guayalejo proviene de la variabilidad genética provocada por irradiación a la semilla de la variedad Mante 81, a la que en 1990 se aplicó una dosis de 18 kilorads y luego se sembró en campo en el ciclo Otoño-Invierno (O-I) 19901991. En los tres ciclos O-I posteriores se aplicó selección masal y de 1994 a 1997 se establecieron pruebas preliminares de rendimiento (surco por planta) para seleccionar las mejores familias. Estas familias se evaluaron en ensayos uniformes regionales, en un diseño experimental de bloques al azar con cuatro repeticiones, durante los ciclos

${ }^{1}$ Aceites Especiales TH. Av. Eje Norte Sur 451 Cd. Industrial Morelia, Mich. México. 58200, Tel +52 (443)204 1032 www.acth.mx
O-I 1997-1998 a 1999-2000. En los tres ciclos 0-I subsecuentes (2000-2001 a 2002-2003) se procedió a la validación interna de las tres mejores familias, de las cuales la familia M81R1M2-S70-S3 pasó a la fase de validación interna-externa. Esta fase se prolongó hasta el ciclo 20102011, en el que se procedió a su caracterización (Cervantes, 2008; Valadez y Montoya, 2011).

Durante el periodo 2004-2007 el rendimiento promedio de la variedad Guayalejo (1,350 kg/ha) superó en $15 \%$ a la variedad Tantoán 91. En el periodo de 2008 al 2012, en evaluaciones efectuadas en localidades del sur de Tamaulipas, el rendimiento de grano de Guayalejo aventajó en $12.79 \%$ a RC-1005-L, en $1.80 \%$ a RC-1033-L y en $16.90 \%$ a CIANO-OL (Cuadro 1), que eran tres variedades generadas en la región noroeste de México. En el ciclo 0-I 20112012 se observó mayor incidencia de Alternaria carthami y Puccinia cartami en las variedades RC-1033-L y CIANO-OL (con valores de 3.4 y 4.6 respectivamente; escala de 0 a 9) que en Guayalejo y RC-1005-L (ambas con 2.6 de calificación) (Cuadro 2).

Entre las principales características agronómicas, destaca su ciclo vegetativo intermedio. En condiciones óptimas de siembra (del 1 al 15 de diciembre), la floración inicia a los 100 d y la madurez fisiológica a los 140 d (Cuadro 3). Presenta una altura promedio de $120 \mathrm{~cm}$ y un hábito de

Cuadro 1. Rendimiento de grano ( $\mathrm{kg} / \mathrm{ha}$ ) de la variedad de cártamo Guayalejo cultivada en seis ambientes de validación, y sus diferencias promedio con respecto a genotipos introducidos del Noroeste de México. CEHUAS-CIRNE-INIFAP.

\begin{tabular}{|c|c|c|c|c|c|c|c|c|}
\hline \multirow[b]{2}{*}{ Genotipo } & 2008-09 & $2009-10$ & $2009-10$ & 2010-11 & $2010-11$ & 2011-12 & \multirow[b]{2}{*}{ Prom. } & \multirow[b]{2}{*}{$\begin{array}{c}\text { Dif. vs. } \\
\text { Guayalejo (\%) }\end{array}$} \\
\hline & $\mathrm{CEHUAS}^{+}$ & CEHUAS & $\begin{array}{l}\text { Col. Campo } \\
\text { Nvo. }{ }^{\ddagger}\end{array}$ & CEHUAS & Tantoyuquita $\ddagger$ & CEHUAS & & \\
\hline Guayalejo & 1920 & 1020 & 775 & 2081 & 1178 & 586 & 1260 & 0 \\
\hline RC-1005-L & 1789 & 1089 & 657 & 1196 & 1196 & 667 & 1099 & -12.79 \\
\hline RC-1033-L & 1700 & 1536 & 1124 & 1556 & 1020 & 488 & 1237 & -1.80 \\
\hline CIANO OL & 1835 & 913 & 588 & 1578 & 1090 & 279 & 1047 & -16.90 \\
\hline
\end{tabular}

${ }^{+}$CEHUAS = Campo Experimental Las Huastecas; ;Localidades del Municipio de González, Tam. 
Cuadro 2. Calificación de incidencia de enfermedades (Alternaria $c^{+*}$. y Puccinia $c^{\ddagger \star *}$.) de cuatro variedades de cártamo, en el ciclo 0-I 2011-2012 en Villa Cuauhtémoc, Tam.

\begin{tabular}{lc}
\hline Genotipo & Calificación en la parte superior el dosel, en escala de 0 al 9 \\
\hline Guayalejo & 2.6 \\
RC-1005-L & 2.6 \\
RC-1033-L & 3.4 \\
CIANO-OL & 4.6 \\
\hline
\end{tabular}

'Mancha de la hoja (Alternaria carthami); ; Roya (Puccinia carthami)

Cuadro 3. Características agronómicas de la variedad de cártamo Guayalejo, comparada con variedades comerciales. CEHUAS-CIRNE-INIFAP. 2007.

\begin{tabular}{|c|c|c|c|c|c|}
\hline \multirow{2}{*}{ Variedad } & \multicolumn{2}{|r|}{ Días a } & \multirow{2}{*}{ Reacción a roya $\left({ }^{\ddagger}\right)$} & \multirow{2}{*}{ Altura de planta $(\mathrm{cm})$} & \multirow{2}{*}{ Peso específico (g/L) } \\
\hline & Floración & Madurez fisiológica & & & \\
\hline Guayalejo & 100 & 140 & 3 & 120 & 566 \\
\hline Tantoán 91 & 96 & 138 & 5 & 115 & 523 \\
\hline Mante 81 & 110 & 140 & 2 & 135 & 550 \\
\hline
\end{tabular}

$\left({ }^{\ddagger}\right) 0$ = resistente; 9 = muy susceptible.

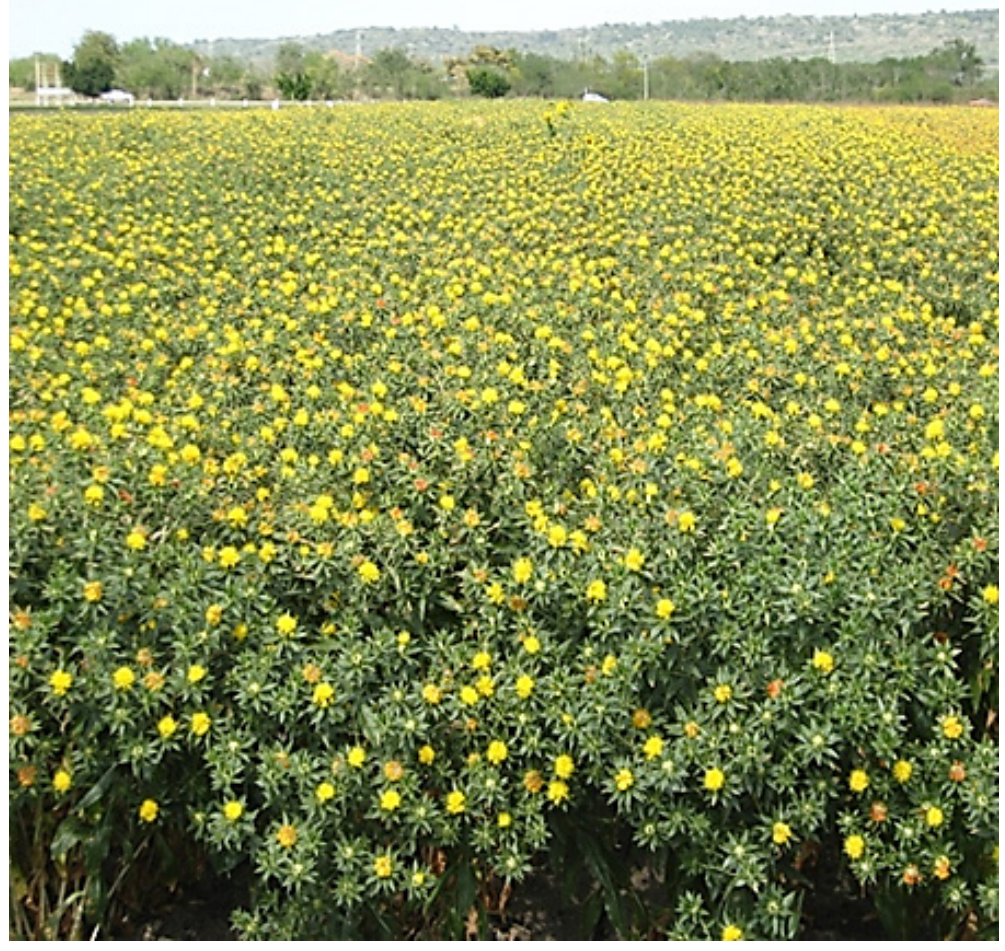

Figura 1. Plantación de cártamo var. Guayalejo en etapa de plena floración. Villa Cuauhtémoc, Tam. Ciclo 0-I, 2009-2010. 
crecimiento determinado y ramificado. Las hojas son de color verde claro, con borde aserrado, de forma angosta en su base y ancha en el ápice. La disposición de las ramas es en ángulo intermedio de 20 a $60^{\circ}$ en los dos tercios superiores de la planta. El tallo es erecto, sólido y resistente al acame.

En promedio la planta presenta 25 capítulos, con 40 granos por capítulo y un diámetro de $2.5 \mathrm{~cm}$ a madurez de cosecha. Las flores frescas son de color amarillo (Figura 1) y al secarse permanecen del mismo color en $60 \%$ de las plantas, mientras que en el resto se tornan rojizas. Lo anterior corresponde a la variabilidad genética que caracteriza a esta variedad. El polen es de color amarillo. Las brácteas son de tamaño medio de forma oval, con ápice agudo y de color verde claro; forman un involucro que cubre el capítulo en su totalidad. Cada bráctea basal presenta un promedio de 15 espinas, que equivale a un alto índice de espinosidad. El grano de Guayalejo es de tamaño medio $(7.3 \mathrm{~mm}$ de longitud y $4.0 \mathrm{~mm}$ de ancho), de forma oval anguloso y de color blanco sucio. Su composición estructural promedio es $35 \%$ de pericarpio y $65 \%$ de almendra, carente de vilano. El peso promedio de 1,000 granos es de $40 \mathrm{~g}$, con un peso específico de $566 \mathrm{~g} / \mathrm{L}$ de semilla ajustado a $7 \%$ de humedad (Cuadro 3).

El área de adaptación para esta nueva variedad de cártamo comprende la región de Las Huastecas, así como regiones del país con condiciones agroclimáticas similares: clima, cálido subhúmedo con lluvias en verano (AW") y semiseco muy cálido y cálido $\left(\mathrm{BS}_{1}\right)$ en condiciones de riego
(INEGI, 1994). Para obtener buenos rendimientos es necesario aplicar las especificaciones descritas en la Guía para Cultivar Cártamo en Las Huastecas (Valadez et al., 2011) y la Guía para Cultivar Cártamo Optimizando Captación de Agua, para Siembras de Temporal en Las Huastecas (Valadez, 2015). En el Campo Experimental Las Huastecas, se dispone de semilla original para producir las categorías básica y registrada que requieran los productores o compañías de semillas interesadas.

\section{BIBLIOGRAFÍA}

Cervantes M. J. E. (2008) Guayalejo, Variedad de Cártamo para el Noreste de México. In: J. Elizondo B. y N. Maldonado M. (comps.). Tecnologías Generadas, Validadas, Transferidas o Adoptadas en el Estado de Tamaulipas en el Periodo 2003-2007. Folleto Técnico No. 40 INIFAP.CIRNE. México. pp:41-42.

INEGI (1994) Anuario Estadístico del Estado de Tamaulipas, Edición 1994. Instituto Nacional de Estadística, Geografía e Informática Aguascalientes, Ags. pp:7-8,11

SNICS, Servicio Nacional de Inspección y Certificación de Semillas (2015) Catálogo Nacional de Variedades Vegetales. No. 7. Secretaría de Agricultura, Ganadería, Desarrollo Rural, Pesca y Alimentación. p.10. http://snics.sagarpa.gob.mx/Documents/2015/CNVV.pdf

Valadez G. J. (2015) Guía para Cultivar Cártamo Optimizando Captación de Agua Para Siembras de Temporal en las Huastecas. Instituto Nacional de Investigaciones Forestales, Agrícolas y Pecuarias. Centro de Investigación Regional del Noreste. Campo Experimental las Huastecas. Villa Cuauhtémoc Tamaulipas, Folleto Técnico No. MX-0-310301-52-03-14-09-40. 25 p.

Valadez G. J. y L. Montoya C. (2011) Rendimiento de -grano de nuevos genotipos de cártamo en parcelas de validación en el sur de Tamaulipas, ciclo Otoño-Invierno 2009-2010. Academia Mexicana Multidisciplinaria 1:7-11.

Valadez G. J., E. Aguirre A., J. C. García R. y J. Ávila V. (2011) Guía para Cultivar Cártamo en Las Huastecas. Centro de Investigación Regional del Noreste, Campo Experimental las Huastecas, Villa Cuauhtémoc, Tamaulipas. Folleto para Productores No.18. 45 p. 